\title{
CONTROL OF LEADING-EDGE VORTICES ON A DELTA WING ${ }^{1}$
}

\author{
by \\ C. Magness, O. Robinson, and D. Rockwell
}

\section{INTRODUCTION}

The unsteady flow structure of leading-edge vortices on a delta wing has been investigated using new types of experimental techniques, in order to provide insight into the consequences of various forms of active control. These investigations involve global control of the entire wing and local control applied at crucial locations on or adjacent to the wing. Transient control having long and short time-scales, relative to the convective time-scale $\mathrm{C} / \mathrm{U}_{\infty}$, allows substantial modification of the unsteady and time-mean flow structure.

Global control at long time-scale involves pitching the wing at rates an order of magnitude lower than the convective time-scale $\mathrm{C} / \mathrm{U}_{\infty}$, but at large amplitudes. The functional form of the pitching maneuver exerts a predominant influence on the trajectory of the feeding sheet, the instantaneous vorticity distribution, and the instantaneous location of vortex breakdown.

Global control at short time-scales of the order of the inherent frequency of the shear layer separating from the leading-edge and the natural frequency of vortex breakdown shows that "resonant" response of the excited shear layer-vortex breakdown system is attainable. The spectral content of the induced disturbance is preserved not only across the entire core of the vortex, but also along the axis of the vortex into the region of vortex breakdown. This unsteady modification results in time-mean alteration of the axial and swirl velocity fields and the location of vortex breakdown.

Localized control at long and short time-scales involves application of various transient forms of suction and blowing using small probes upstream and downstream of the location of vortex breakdown, as well as distributed suction and blowing along the leading-edge of the wing applied in a direction tangential to the feeding sheet. These local control techniques can result in substantial alteration of the location of vortex breakdown; in some cases, it is possible to accomplish this without net mass addition to the flow field.

\section{EXPERIMENTAL TECHNIQUES}

The unsteady flow structure from the leading-edge of a delta wing subjected to various forms of active control has been characterized using new types of laser-diagnostic systems and image-processing techniques. These methods are integrated with active control systems, driven by central microcomputers. Using these approaches, it is possible to impose active control of arbitrary functional form and examine the response of the instantaneous flow structure. The two- and three-dimensional flow structure is interpreted with the aid of newly-released graphics supercomputers.

\section{GLOBAL CONTROL AT LONG TIME SCALES}

The concept of a phase shift between the unsteady motion of the wing and the development of the leading-edge vortex is well known. In qualitative visualization studies, Lambourne et al. (1969), Gad-el-Hak and Ho (1985a,b, 1986), and Atta and Rockwell (1989) reveal various features of the visualized cross-section of the vortex during its unsteady

\footnotetext{
${ }^{1}$ Submitted for presentation at the NASA/AFOSR/ARO Workshop on Physics of
} Forced Separation, April 17-19, 1990. 
development. There also occurs a phase shift of the location of vortex breakdown relative to the wing motion; it has been characterized from various perspectives by Woffelt (1986), Rockwell et al. (1987), Atta and Rockwell (1987), Reynolds and Abtahi (1987), Gilliam, Robinson, Walker, Wisser (1987), and Lemay, Batill, and Nelson (1988).

The following unresolved issues are the focus of this investigation: the effect of arbitrary forms of pitching maneuver on the instantaneous structure of the leading-edge vortex including trajectories of feeding sheets and distributions of vorticity; the influence of vortex breakdown over a portion of the cross-section of the vortex; and the response of the axial location of vortex breakdown in relation to all of these features.

Concerning the nature of the instantaneous structure of the leading-edge vortices, obtained from particle tracking techniques, the following represent the major findings:

(i) For locations upsteam of vortex breakdown, the shape, degree of concentration, and the location of the maxima of the instantaneous vorticity distribution across the vortex core are quite different for the up- and downstrokes of the continuous pitch-up-down maneuvers of the wing. This finding emphasizes the importance of accounting for the instantaneous cross-sectional structure of the vortex, and not simply the instantaneous location of vortex breakdown, in determining the overall loading on the wing.

(ii) Comparison of the vorticity distribution of the leading-edge vortex with the trajectory of the feeding sheet from the edge of the wing shows the relationship between the possible trajectories of the feeding sheet and the corresponding vorticity field. A major factor is the occurrence or non-occurrence of vortex breakdown within the core of the vortex.

Figure 1 shows an excerpt from the current investigation. Contours of constant vorticity were obtained by direct particle tracking and image processing techniques. The experimental parameters correspond to a pitching motion of $15^{\circ} \leq \alpha \leq 40^{\circ}$ for a continuous pitch-up-down motion at a pitching rate $\dot{\alpha} \mathrm{C} / 2 \mathrm{U}_{\infty}=0.15$. The surface of the wing is indicated by the bold horizontal line. The contours of constant vorticity on the left side correspond to the pitch-up portion of the maneuver, and those on the right side to the pitchdown portion. The differences in elevation, orientation, and scale of the vorticity distributions are evident. They are dependent upon the history of the wing motion and appear to be most pronounced at the smallest angle of attack $\alpha=20^{\circ}$.

The importance of accounting for vortex breakdown within the core of the vortex is illustrated in Figure 2. Instantaneous positions of the feeding sheets and contours of constant vorticity are shown for the same parameters as in Figure 2, but at $\alpha=40^{\circ}$ for two different types of maneuvers. The shaded black region represents the extent of breakdown within the vortex. For the simple pitch-down motion, $\alpha=40^{\circ}$ represents the static condition immediately preceding the onset of the maneuver, while the pitch-up-down case at $\alpha=40^{\circ}$ includes the integrated history of the upstroke portion of the maneuver. It is evident that the positions of the feeding sheet and the contours of vorticity are substantially different for these two cases.

The structure of the leading-edge vortex at a given cross-section must, of course, be considered in conjunction with the axial movements of the location of vortex breakdown. Magness, Robinson, and Rockwell (1989) preliminarily addressed the effect of the type of pitching maneuver on the general response of the vortex breakdown location as a function of angle of attack. Recent studies have focussed on the vortex response to different classes of maneuver, and the detailed structure of the leading-edge vortices. Regarding the response of the location of the vortex breakdown, the major findings are: 
(i) Continuous pitch up-down motions of the wing can preclude occurrence of vortex breakdown on the upstream portion of the wing, relative to that occurring for simple pitch-up and pitch-down motion where relaxation processes baving long time scales are allowed to occur.

(ii) For the continuous pitch-up-down maneuver of the wing, the consequence of not allowing the vortex breakdown to relax to its equilibrium state is to produce upstream movements of the vortex breakdown location towards the apex for initial decreases in angle of attack $\alpha$.

(iii) Combinations of simple ramp-type motions to form a hybrid pitching motion produce overshoots of the static characteristic of vortex breakdown location versus angle of attack, beyond that attainable with any of the simple ramp motions alone.

Figure 3 shows plots characterizing the first two of these three principal findings at two extreme values of reduced frequency. This sort of characterization of the breakdown location serves as a basis for detailed investigations of the flow structure of the leading-edge vortices.

\section{GLOBAL CONTROL AT SHORT TIME SCALES}

Perturbation of a delta wing in the pitching mode at sufficiently high frequency and very low amplitude allows control of the detailed flow structure of the leading-edge vortex. In essence, the vortex development and breakdown on a delta wing involves two classes of characteristic frequencies: the inherent instability frequency of the shear layer from the leading-edge; and the frequency at which vortex breakdown occurs. The major issues here are: the structure of the perturbed feeding sheet; the nature of the perturbed onset of vortex breakdown; and the corresponding alteration of the time-mean vortex flow.

Simple considerations of hydrodynamic instability show that the processes of disturbance amplification in the shear layer and in the vortex core during the breakdown process are receptive to a wide range of excitation frequencies. As a consequence, it is possible to attain "resonant" excitation, leading to large alteration of the separating shear layer from the edge of the wing and the breakdown of the vortex core. The preliminary phase of this investigation was reported by Rockwell et al. (1987). This work is described in its completed form by Kuo, Magness, and Rockwell (1989).

The principal findings of this investigation are, in short:

(i) Small amplitude perturbations of the leading-edge lead to substantial alteration of the structure of the shear layer separating from it without occurrence of the classical mechanism of small-scale vortex coalescence.

(ii) The spectral content of the disturbance induced in the shear layer separating from the leading-edge is preserved not only across the core of the vortex, but also along the streamwise extent of the core into the region of vortex breakdown.

(iii) Substantial alteration of the time-mean characteristics of the leading-edge vortex include changes in the axial and swirl velocity fields and modification of the location of vortex breakdown.

Selected excerpts describing certain of the foregoing phenomena are given in Figures 4 through 6 . Figure 4 shows the visualization obtained by locating a vertical hydrogen bubble wire along the leading-edge of the wing. The laser sheet that illuminated the marker bubbles was translated to the downstream locations $\mathrm{x} / \mathrm{C}$ indicated in the photos. Excitation frequency 
$f_{e}$ is normalized with respect to the inherent instability frequency $f_{i}$ of the shear layer separating from the leading-edge. Large-scale vortical structures are induced over the crosssection of the vortex in the presence of excitation at the inherent instability frequency of the feeding sheet. No small-scale vortex coalescence occurs.

Figure 5 shows spectra of the streamwise component $\tilde{u}$, i.e. $S_{\tilde{u}}$, taken at various locations upstream and downstream of the onset of vortex breakdown. The edge excitation frequency $f_{e}$ is normalized by the inherent vortex breakdown frequency $f_{b}$. For excitation at the first harmonic of the vortex breakdown frequency, i.e. at $f_{e} / f_{b}=2$, the spectral content shows predominance of the excitation frequency and its associated higher harmonics in regions before and after occurrence of vortex breakdown. In this case, the higher harmonic content persists well downstream of the onset of breakdown. For excitation at $f_{e} / f_{b}=1$, there also occur a large number of higher harmonics due to the strong nonlinearity of the shear layer response. This spectral content is maintained over the entire cross-section of the vortex core prior to the occurence of breakdown, emphasizing the nonoccurence of vortex-vortex interactions (i.e. coalescence) in the shear layer as it is wrapped inwards toward the center of the core. Downstream of vortex breakdown, the predominant excitation peak at $f_{e} / f_{b}=1$ persists, but the coherent higher harmonic components are attenuated.

Figure 6 shows contours of constant mean axial velocity $\bar{u}$ and constant fluctuating velocity $\tilde{u}$ over the entire cross-section of the leading-edge vortex at values of excitation frequency $f_{e}$, relative to the inherent vortex breakdown frequency $f_{b}$, i.e. $f_{e} / f_{b}=1$ (left column) and 2 (right column). The effect of the matched excitation at $f_{e} / f_{b}=1$ is to induce large amplitude fluctuations in the separating shear layer surrounding the core of the vortex, located at the peak of the contours of constant $\bar{u}$ and designated by the symbol + . At $f_{e} / f_{b}=2$, the location of the core of the vortex moves downward towards the surface of the wing and outward towards the leading-edge. The maximum amplitude of the fluctuation $\tilde{\mathbf{u}}$ is coincident with the location of the core of the vortex. This coincidence of the maxima of $\bar{u}$ and $\tilde{u}$ corresponds to the early onset of vortex breakdown at the higher excitation frequency $f_{e} / f_{b}=2$.

\section{LOCAL CONTROL AT MODERATE AND LONG TIME SCALES}

Local control involves localized injection or suction of the flow at defined locations in the flow field and/or the surface of the wing. In a practical sense, this can be achieved by use of small probes, whose tips are located at crucial locations in the vortex core, or slits along the leading-edge of the wing. In essence, these techniques simulate localized point sources/sinks or distributions of them. The major issues here are: determination of the most sensitive location of the applied control; and optimization of the functional form of the unsteady control in the form of blowing/suction.

For the case of localized blowing along the leading-edge of the wing, Wisser, Iwanski, Nielson, and $\mathrm{Ng}$ (1988) most recently have revealed an increase in length of the vortex core prior to breakdown and an increase in lift acting on the wing. Not until this past year has the case of localized suction been explored; such simulations of a localized sink are described by Parmenter and Rockwell (1989). Location of a probe in the region downstream of vortex breakdown allows efficient restabilization of the vortex core. Among the principal findings are:

(i) Locations of the simulated point sink downstream of the occurrence of vortex breakdown produces stabilization of the core; such stabilization is attainable at relatively low values of dimensionless suction coefficient $C_{\mu}$. The transient response time of the stabilization process due to an imposed transient (unsteady sink flow) scales as the magnitude of the imposed transient suction.

(ii) Hysteresis effects occur due to relaxation of the vortex breakdown (on a stationary wing) after abrupt onset or cessation of suction. These hysteresis effects simulate those on a pitching delta wing. 
Localized control involving simulations of distributed sources/sinks in the form of a blowing/suction slit along the leading-edge have received little attention except for the steady blowing experiments of Wood and Roberts (1987), Wood, Roberts and Lee (1987) and Roberts, Hesselink, Kroo, and Woods (1987), and the (high frequency) sinusoidal perturbations employed in the investigation of Gad-el-Hak and Blackwelder (1987). The consequences of this type of control on the structure of the large-scale vortex have remained unexplored. Moreover, the possible modification of the nature and location of onset of vortex breakdown has not been pursued. Important considerations in our recent investigations include not only the case of steady blowing, but also the corresponding case of steady suction and, most significantly, the case of cyclic blowing and suction. The major findings of this investigation are:

(i) Both steady suction and steady blowing are effective at low values of $\mathrm{C}_{\mu}$, i.e. both result in lengthening of the vortex core prior to the onset of breakdown.

(ii) The most effective and robust control involves cyclic suction and blowing at an appropriate frequency. This approach involves no net mass addition to or from the flow.

The use of cyclic blowing and suction applied tangentially in the form of a jet $V_{j}(t)$ at the rounded leading-edge is represented in Figure 7 ; it is compared with the case of no blowing/suction, i.e. $V_{j}(t)=0$. (These data were acquired by Professor $W$. Gu, a member of our research group.) Comparison of these velocity fields of Figure 7 suggests that application of the control results in restabilization of the vortex from a stalled condition to a well-defined, large-scale vortical structure and downward deflection of the separation streamline from its approximately horizontal position. These trends are associated with downstream movement of the location of vortex breakdown.

\section{ACKNOWLEDGEMENTS}

The authors gratefully acknowledge support of the Air Force Office of Scientific Research, as part of a program monitored by Captain H. Helin.

\section{LIST OF REFERENCES}

Atta, R. and Rockwell, D. 1987 "Hysteresis of Vortex Development and Breakdown on an Oscillating Delta Wing”, AIAA Journal, Vol. 25, No. 11, pp. 1512-1513.

Atta, R. and Rockwell, D. 1989 "Leading-Edge Vortices Due to Low Reynolds Number Flow Past a Pitching Delta Wing", AIAA Journal (in press).

Gad-el-Hak, M. and Blackwelder, R. F. 1987 "Control of the Discrete Vortices from a Delta Wing", AIAA Journal, Vol. 25, No. 8, pp. 1042-1049.

Gad-el-Hak, M. and Ho, C.-M. 1985a "The Pitching Delta Wing", AIAA Journal, Vol. 23, No. 11, pp. 1660-1665.

Gad-el-Hak, M. and Ho, C.-M. 1985b "Three-Dimensional Effects on a Pitching Lifting Surface", AIAA Paper No. 85-0041, presented at the AIAA 23rd Aerospace Sciences Meeting, January 14-17, Reno, Nevada.

Gad-el-Hak, M. and Ho, C.-M. 1986 "Unsteady Vortical Flow Around Three-Dimensional Lifting Surfaces", AIAA Journal, Vol. 24, No. 5, May, pp. 713-721. 
Gilliam, F., Robinson, N., Walker, J., and Wissler, J. 1987 "Visualization of Unsteady Separated Flow About a Pitching Delta Wing", AIAA 25th Aerospace Sciences Meeting, January 12-15, Reno, Nevada.

Kuo, C.-H., Magness, C., and Rockwell, D. 1989 "Control of Vortex Structure on a Delta Wing by Small-Amplitude Perturbations of Angle-of-Attack", submitted for publication.

Lambourne, N. C., Bryer, D. W., and Maybrey, J. F. N. 1969 "The Behavior of Leading-Edge Vortices Over a Delta Wing Following Sudden Change of Incidencen, The Aeronautical Research Council Technical Report, Report and Memorandum 3645.

LeMay, S. P., Batill, S. M., and Nelson, R. C. 1988 "Leading-Edge Vortex Dynamics on a Pitching Delta Wing", AIAA Paper No. AIAA-88-2559-CP, AIAA Sixth Applied Aerodynamics Conference, June 6-8, Williamsburg, Virginia.

Magness, C., Robinson, O., and Rockwell, D. 1989 "Control of Leading-Edge Vortices on a Delta Wing", AIAA Paper No. 89-0999, presented at AIAA 2nd Shear Flow Conference, March 13-16, Tempe, Arizona.

Parmenter, K. and Rockwell, D. 1989 "Response of Leading-Edge Vortices to Localized Suction", AIAA Journal (in press).

Reynolds, G. A. and Abtahi, A. A. 1987 "Instabilities in Leading-Edge Vortex Development", AIAA Paper No. 87-2424, AIAA Applied Aerodynamics and Atmospheric Flight Dynamics Conference, August 17-19, Monterey, California.

Roberts, L., Hesselink, L., Kroo, I., and Wood, N. 1987 "The Control of Vortical Flows Over a Delta Wing", Proceedings of Workshop on Unsteady Separated Flows at U. S. Air Force Academy. Also see Frank J. Seiler Research Laboratory Report FJSRL-TR-88-0004, September 1988, Air Force Systems Command, United States Air Force.

Rockwell, D., Atta, R., Kuo, C.-H., Hefele, C., Magness, C., and Utsch, T. 1987 "On Unsteady Flow Structure from Swept Edges Subjected to Controlled Motion", Proceedings of Workshop on Unsteady Separated Flows at U. S. Air Force Academy. Also see Frank J. Seiler Research Laboratory Report FJSRL-TR-88-0004, September 1988, Air Force Systems Command, United States Air Force.

Wisser, K., Iwanski, K. T., Nelson, R. C. and Ng, T. T. $1988{ }^{~ " C o n t r o l ~ o f ~ L e a d i n g-E d g e ~}$ Vortex Breakdown by Blowing", AIAA Paper No. 88-0504, AIAA 26th Aerospace Sciences Meeting, January, Reno, Nevada.

Wolffelt, K. W. 1986 "Investigation on the Movement of Vortex Burst Position with Dynamically Changing Angle of Attack for a Schematic Delta Wing in a Water Tunnel with Correlation to Similar Studies in Wind Tunnel", AGARD Conference Proceedings \#413, Aerodynamic and Related Hydrodynamic Studies Using Water Facilities, Symposium of the Fluid Dynamics Panel, Monterey, California, 20-23 October, 1986.

Wood, N. J. and Roberts, L. 1987 “The Control of Vortical Lift on Delta Wings by Tangential Leading-Edge Blowing", AIAA Paper 87-0158, January.

Wood, N. J., Roberts, L., and Lee, K. T. 1987 "The Control of Vortical Flow on a Delta Wing at High Angles of Attack", AIAA Paper 87-2278, August. 


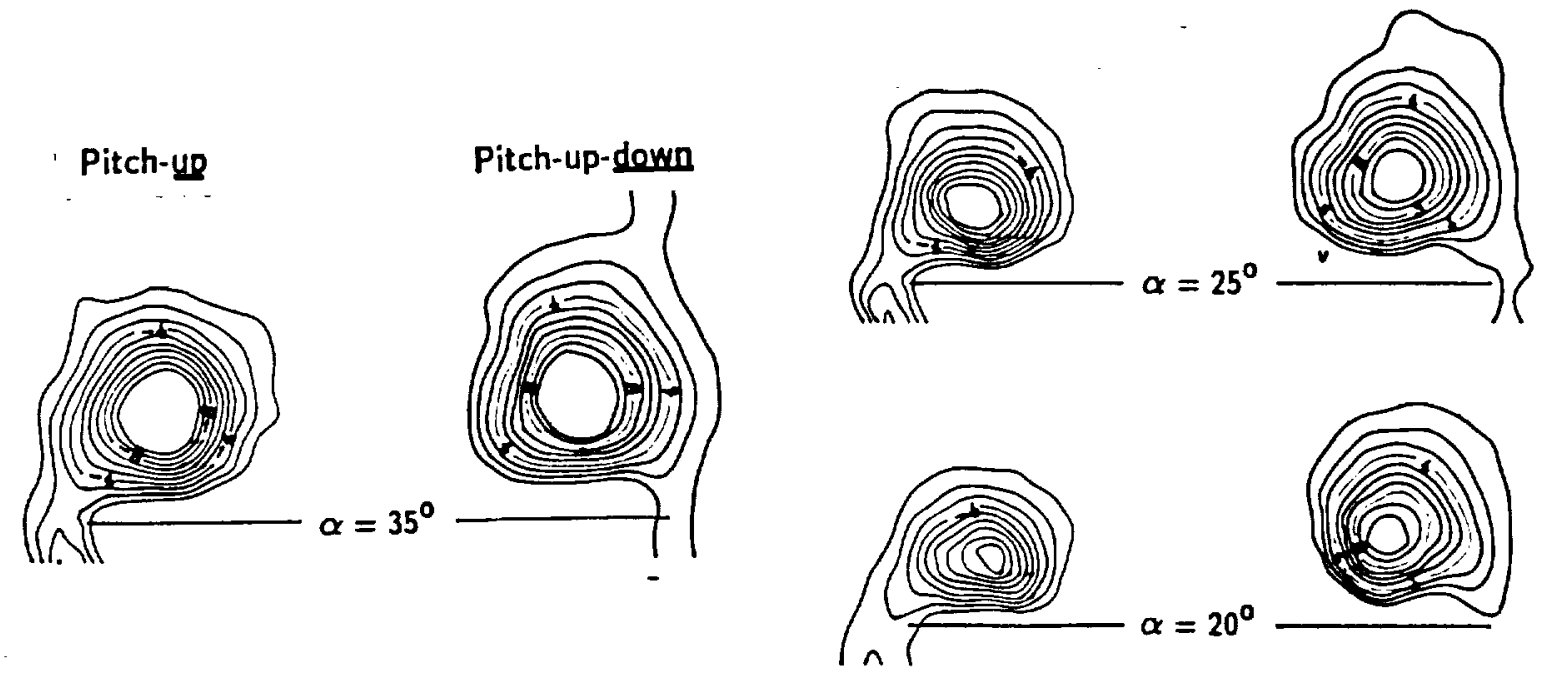

Figure 1: Instantaneous contours of constant vorticity at midchord for continuous pitch-updown maneuver of delta wing. Sweep angle $=75^{\circ}$; pitch rate $\dot{\alpha} C / 2 U=0.15$; pitching axis at midchord.

Pitch-up-down

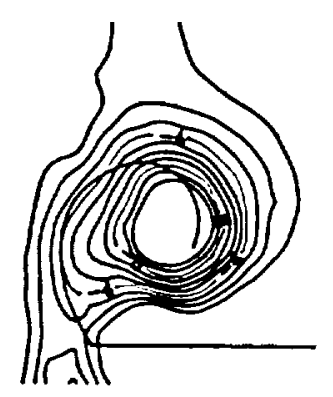

Pitch-down

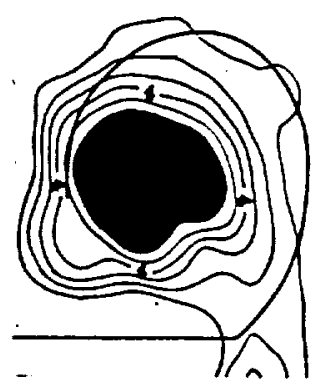

$\alpha=40^{\circ}$

Figure 2: Instantaneous contours of constant vorticity and positions of feeding sheet at $\alpha=40^{\circ}$ for continuous pitch-up-down and pitch-down maneuvers. Sweep angle $=75^{\circ}$; pitch rate $\dot{\alpha} \mathrm{C} / 2 \mathrm{U}=0.5$; pitching axis at midchord. 

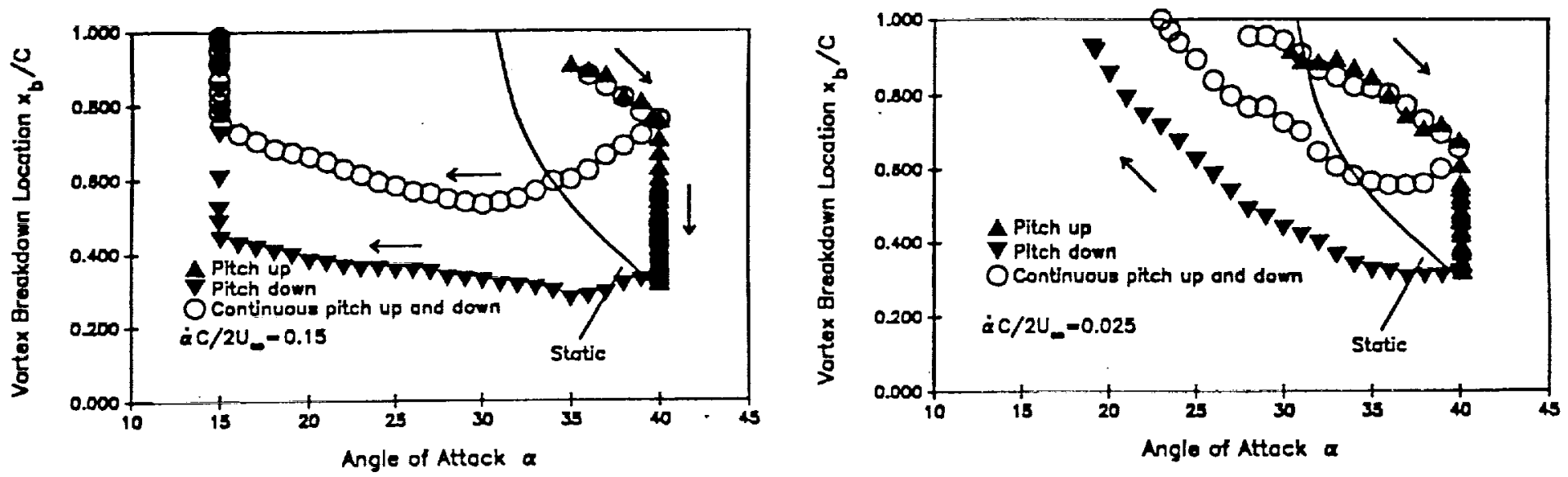

Figure 3: Instantaneous location of vortex breakdown as function of angle of attack for three basic types of delta wing maneuvers and two extreme values of dimensionless pitching rate. Sweep angle of delta wing $=75^{\circ}$.

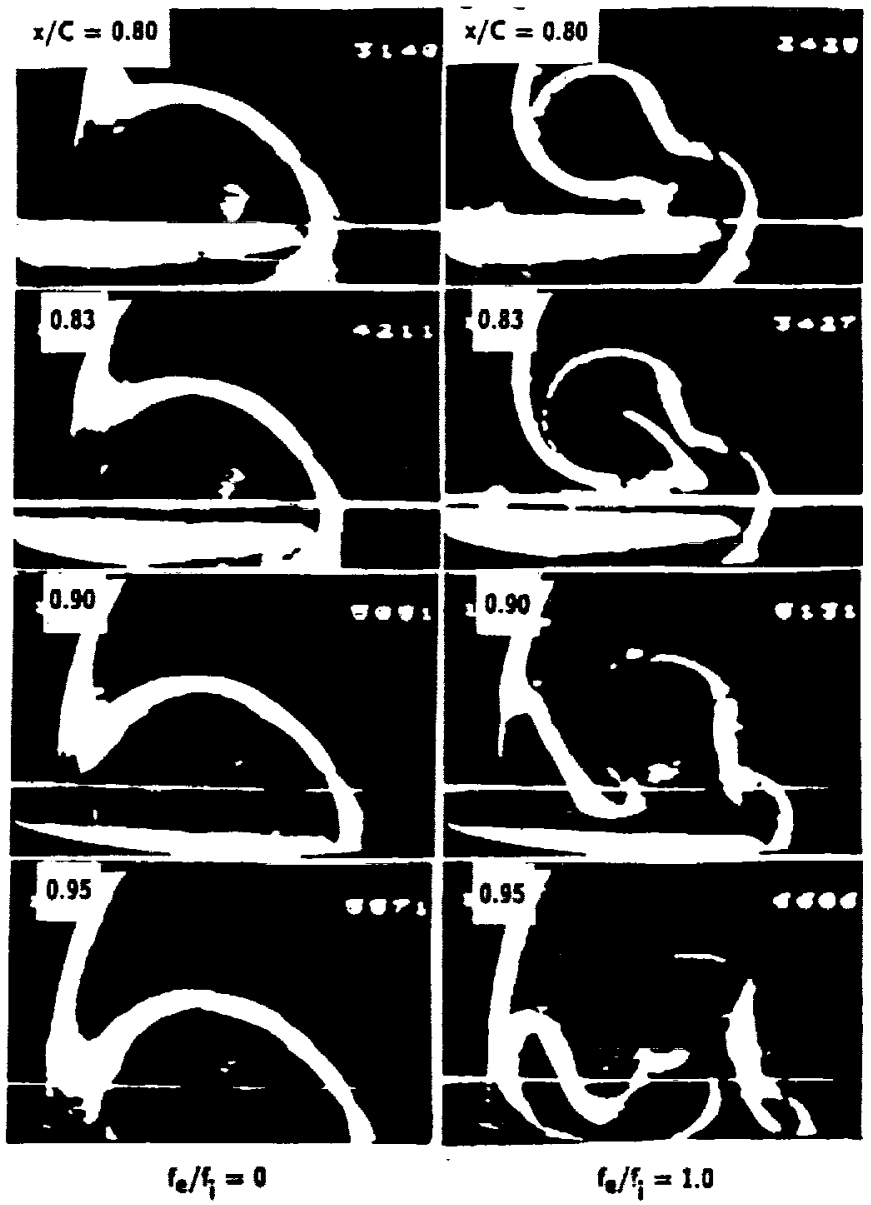

Figure 4: Visualization of flow structure of separating shear layer at several cross-sections along leading-edge of delta wing. Ratio of excitation frequency $f_{e}$ to inherent instability frequency $f_{i}$ of separating shear layer is: 0 (left column); 0.5 (middle column); and 1.0 (right column). Angle of attack $\alpha=\bar{\alpha}+\alpha_{0} \sin 2 \pi \mathrm{f}_{\mathrm{e}} \mathrm{t} ; \bar{\alpha}=20^{\circ}, \alpha_{0}=1^{\circ}$. 


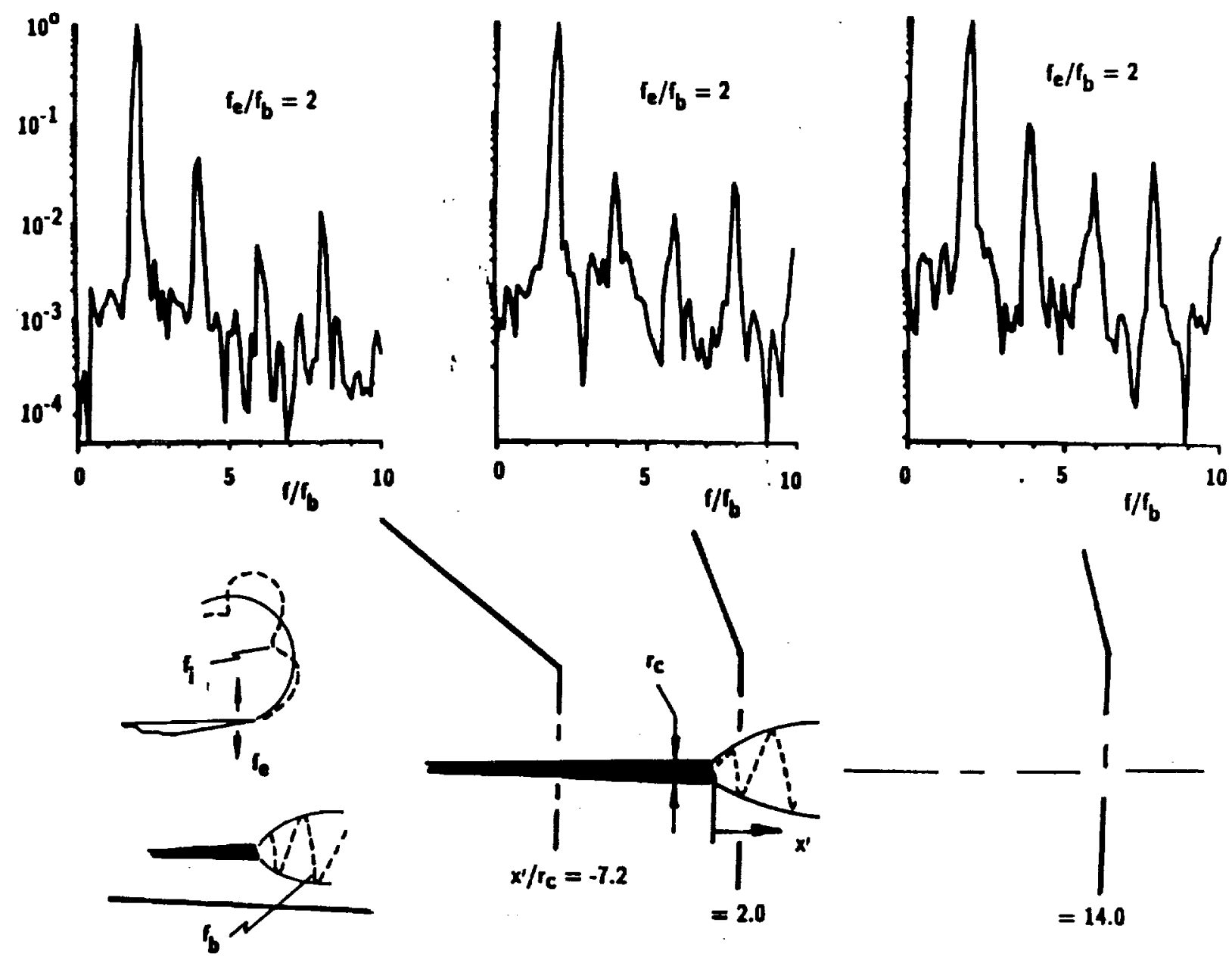

Figure 5: Overview of evolution of spectral content of vortex core upstream and downstream of vortex breakdown. Ratio of excitation frequency $f_{e}$ to vortex breakdown frequency $f_{b}$ has values indicated. $f_{i} / f_{b}=2$. 

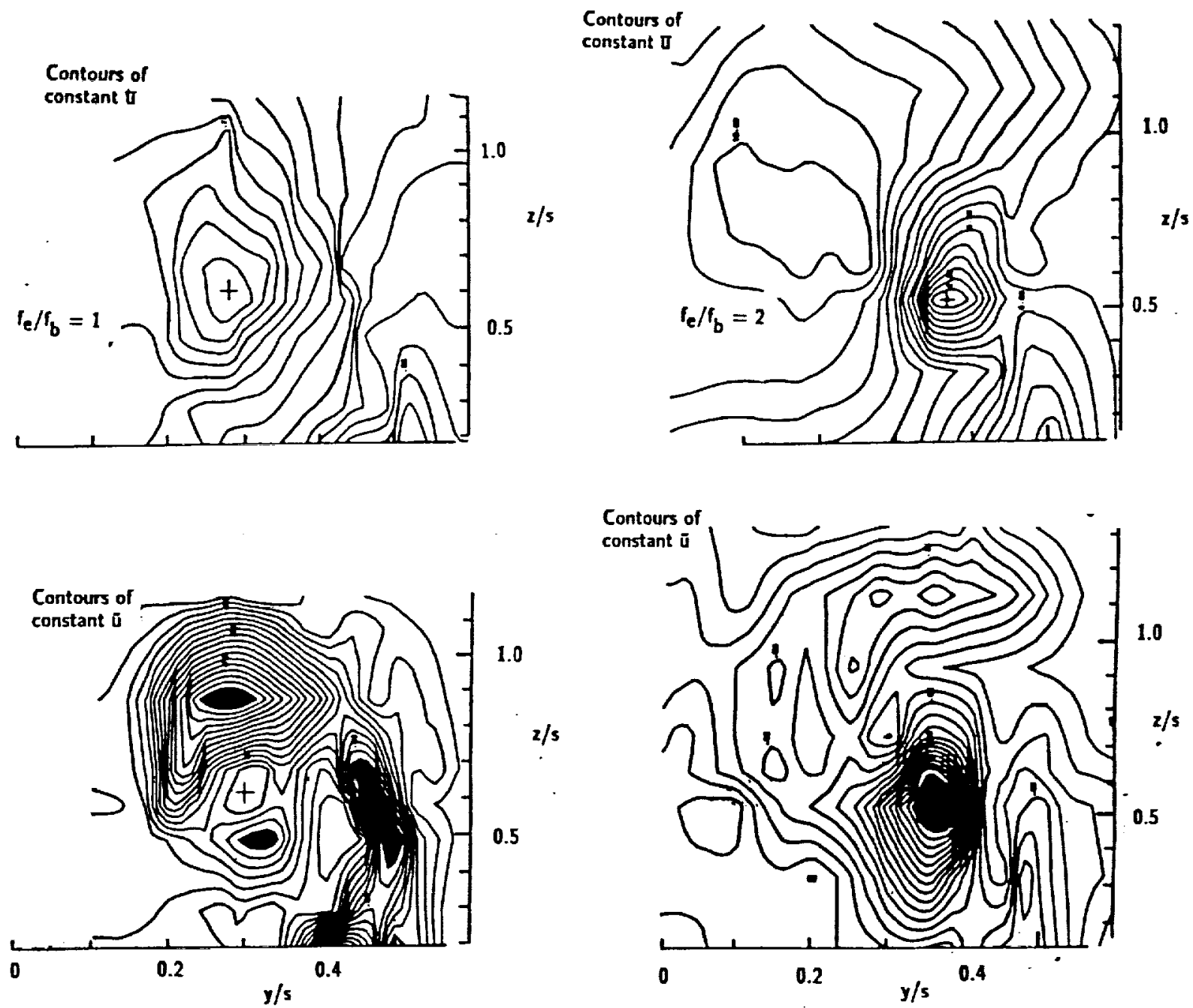

Figure 6: Distribution of mean $\bar{u}$ component of axial velocity over cross-section of vortex upstream of occurrence of vortex breakdown. Data acquired at reference station $x / C=0.38$ upstream of vortex breakdown. Ratio of excitation frequency $f_{e}$ to frequency $f_{b}$ of inherent vortex breakdown is $f_{e} / f_{b}=1$ (left column) and $f_{e} / f_{b}=2$ (right column). Ratio of inherent instability frequency $f_{i}$ of separating shear layer to frequency $f_{b}$ is $f_{j} / f_{b}=2$.
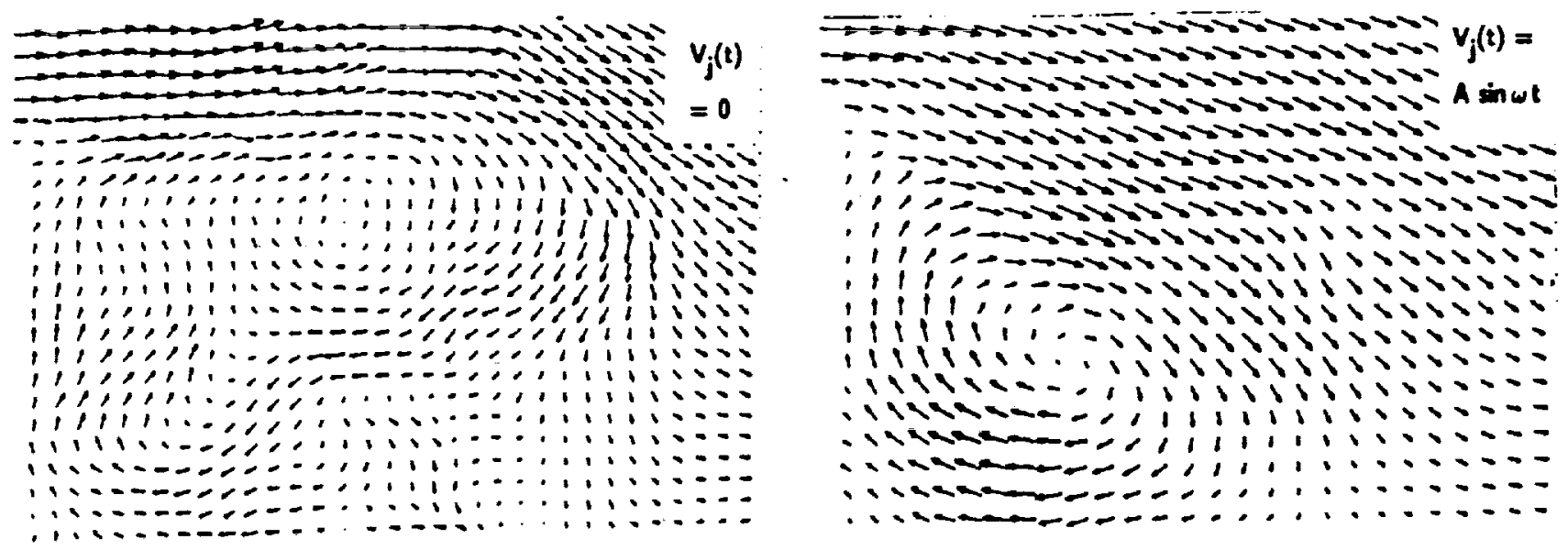

Figure 7: Comparison of velocity fields over cross-section of half delta wing at $\alpha=45^{\circ}$ for cases with and without sinusoidal suction/blowing applied tangentially at rounded leadingedge. Laser sheet defining cross-section of visualized vortex located at approximately one-third chord. 


\section{OVERVIEW}

- Concepts of Control: Time-Scales and Vorticity Budgets

- Experimental Approaches

- Global Control at Small Time-Scales $\mathbf{t U} / \mathbf{C}<<1$

- Global Control at Large Time-Scales $\mathrm{tU} / \mathrm{C}>\mathrm{P}$

- Local Control at Moderate Time-Scales $\mathrm{tU} / \mathrm{C} \sim 1$ 


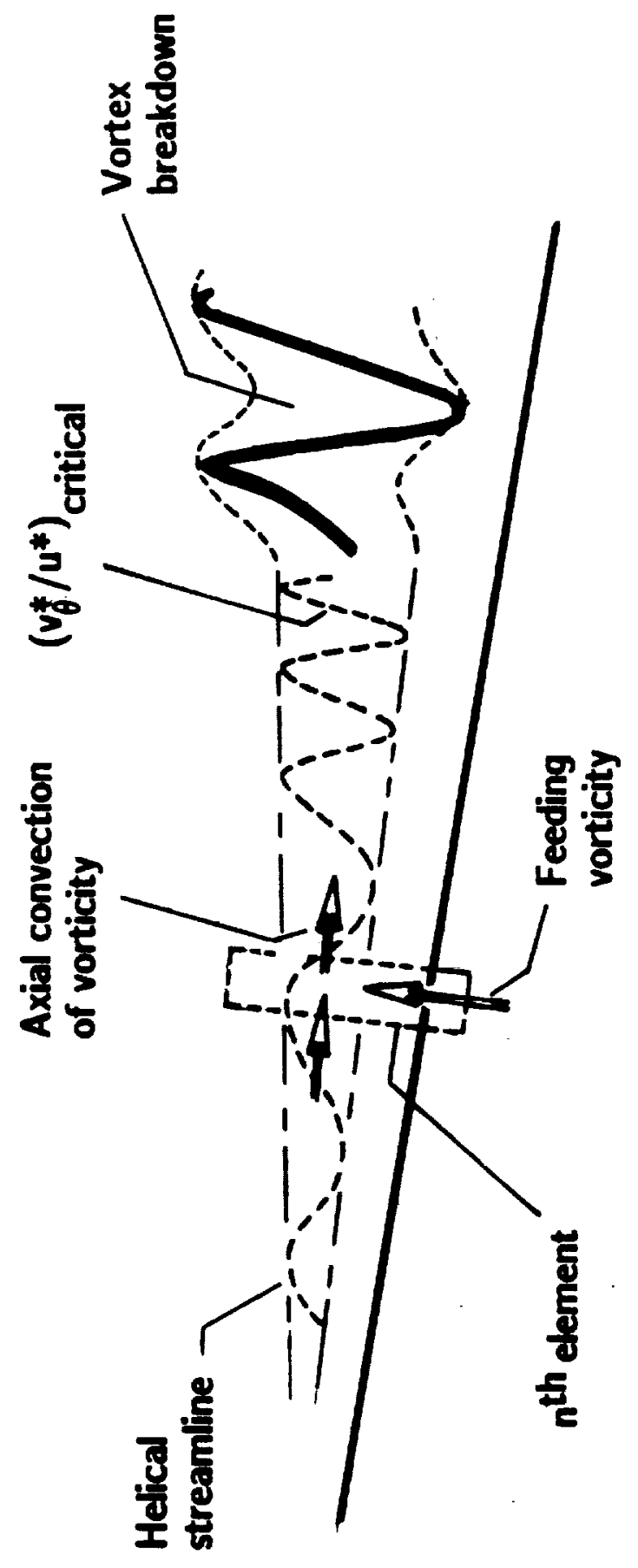


SEPARATION/SEPARATED PHENOMENA
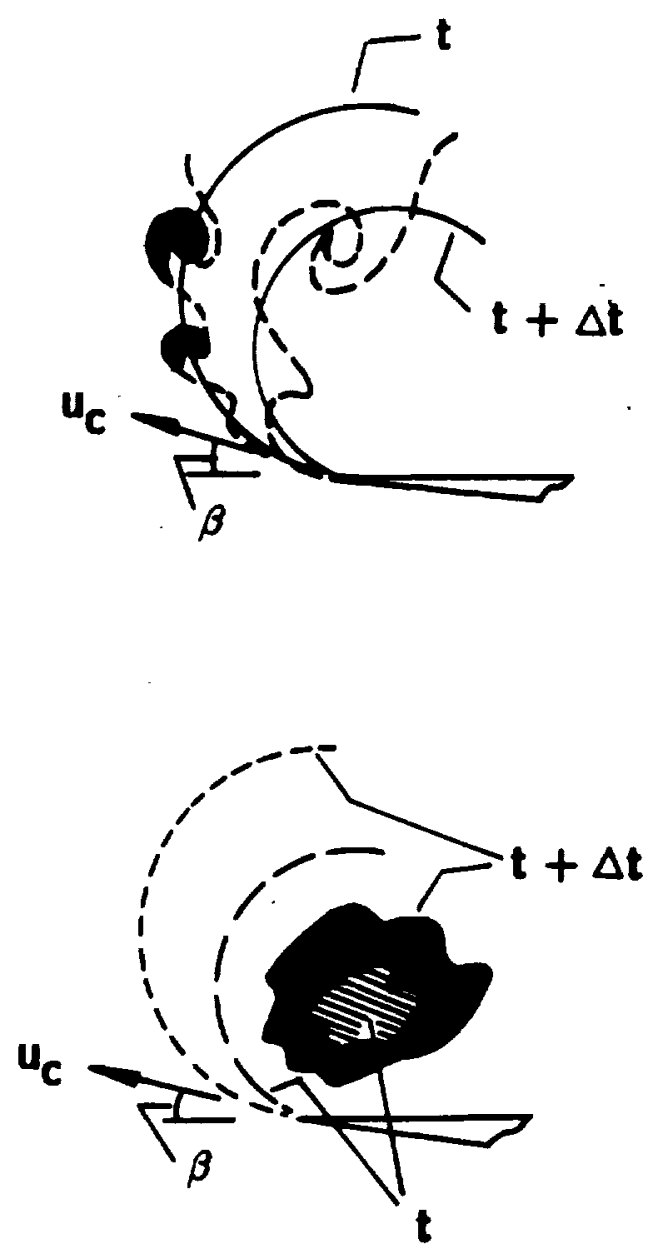

\section{PRINCIPAL MECHANISMS}

Alteration of unsteady concentrations of vorticity of feeding sheet

$$
\begin{gathered}
10^{-2} \mathrm{C} / \mathrm{U} \\
\text { and } \\
\mathrm{C} / \mathrm{U}
\end{gathered}
$$

Alteration of axial convection of vorticity due to vortex breakdown

10 to $10^{2} \mathrm{C} / \mathrm{U}$ 


\section{SEPARATION/SEPARATED PHENOMENA}
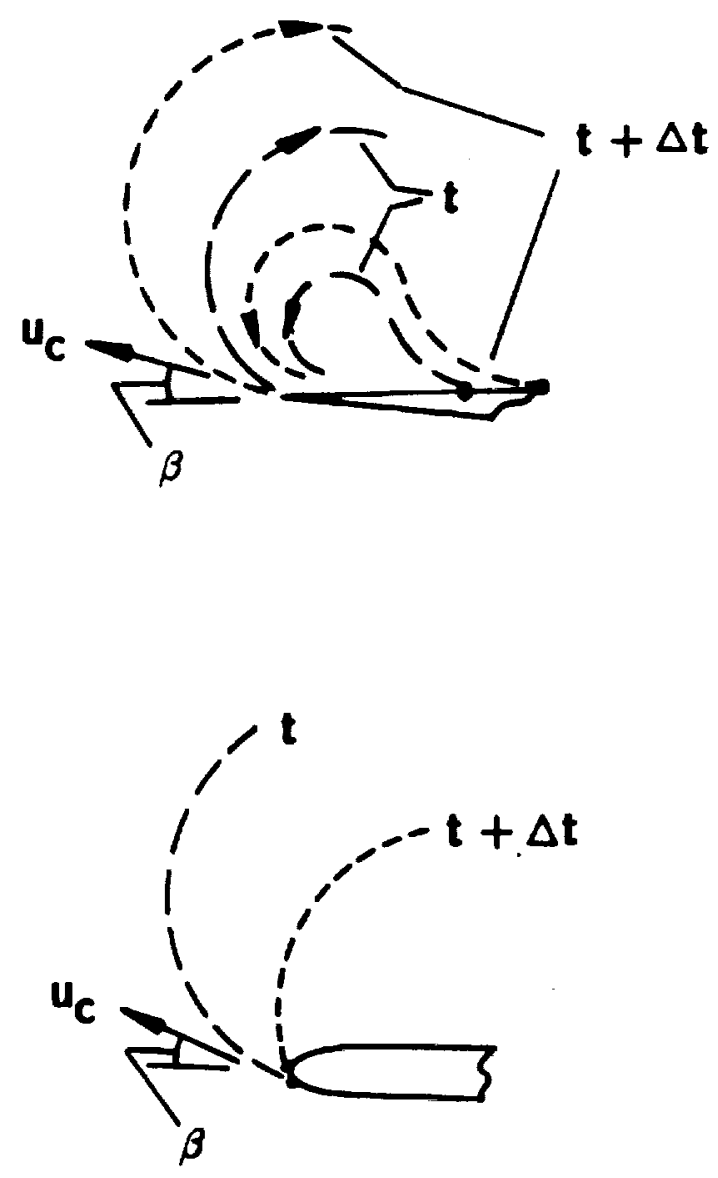

\section{PRINCIPAL MECHANISMS}

Formation of vortex fed by vorticity from leeward surface

$$
\text { C/U }
$$

Movement of separation point of vorticity feeding sheet

$$
\mathrm{C} / \mathrm{U}
$$




\section{EXPERIMENTAL APPROACHES}

- Quantitative Flow Visualization: Global Velocity Measurements

$\checkmark$ Bubble Marker Tracking

$\checkmark$ Particle Tracking

$\checkmark$ Particle Imaging

- Quantitative Flow Visualization: Construction of Two- and Three-Dimensional Images

- Local Velocity Measurement: Laser-Doppler Anemometry

- Force and Pressure Measurements 


\section{EXPERIMENTAL APPROACHES}

- Integrated Active Control

$\checkmark$ Centralized Computer Control of

a Wing Motion (Global);

Blowing/Suction (Local)

口 Laser Firing

口 Image Shifting System

- Camera(s)

- Data Acquisition Systems

$\checkmark$ Arbitrary Functional Forms of Global and Active Control 


\section{GLOBAL CONTROL AT SMALL TIME SCALES tU $/ \mathrm{C}<<1$}

- Forced Instability and Concentration of Vorticity in Feeding Sheet

- Resonant Interaction of Instabilities of Feeding Sheet and Vortex Breakdown

- Preservation of Spectral Content Throughout LeadingEdge Vortex

- Modification of Time-Mean Axial and Swirl Velocity Components 


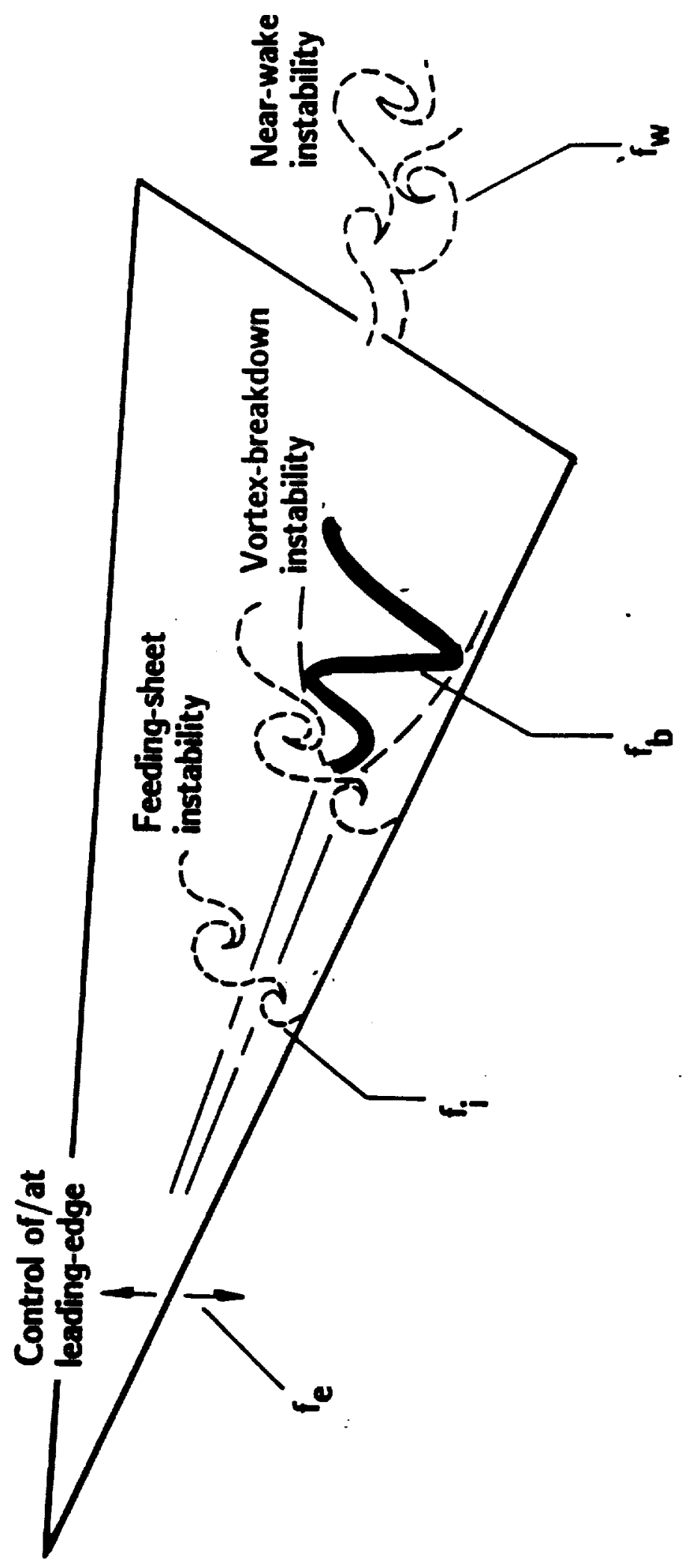

96 


\section{GLOBAL CONTROL AT MODERATE AND LARGE TIME SCALES $\mathrm{tU} / \mathrm{C} \sim 1$ or $>1$}

- Response of Leading-Edge Vortex in Absence of Vortex Breakdown: Sensitivity to Integrated History of Motion

- Response of Leading-Edge Vortex in Presence of Vortex Breakdown: Nonlinear Coupling of Feeding Sheet-Vortex Breakdown-Stall Zone

- Response of Vortex Breakdown: Sensitivity to Class of Forcing

- Response of Feeding Sheets and Vorticity Distributions with and without Vortex Breakdown: Sensitivity to Class of Forcing 


\section{Pitch-up}
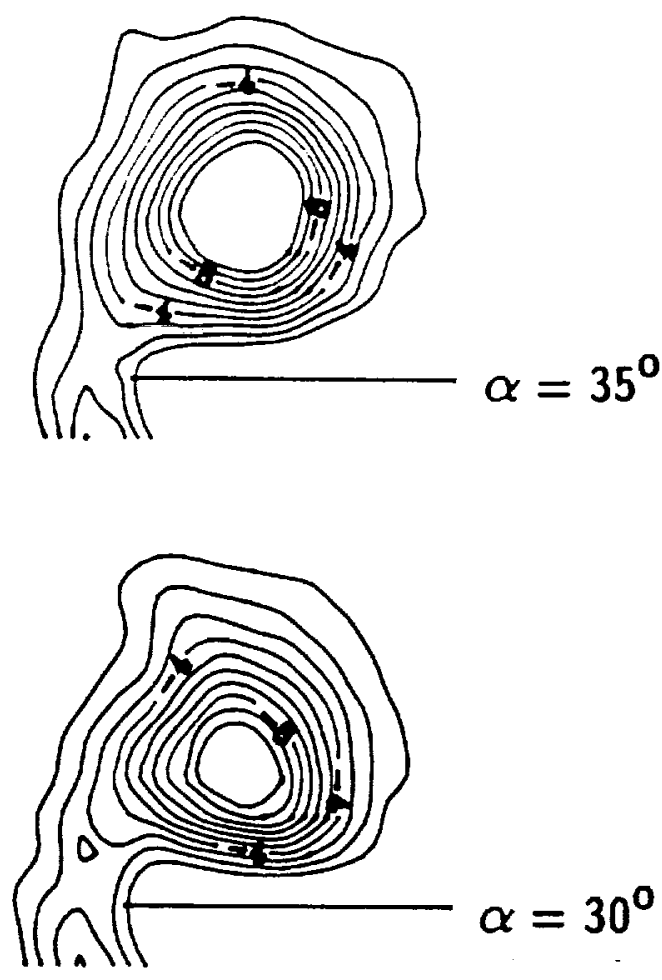

$\alpha=30^{\circ}$
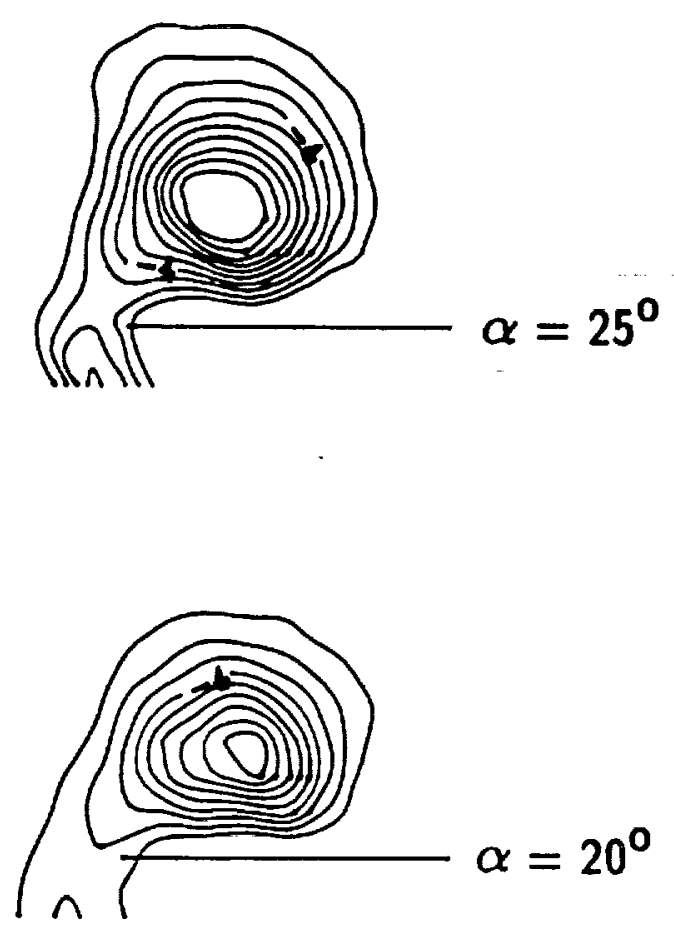
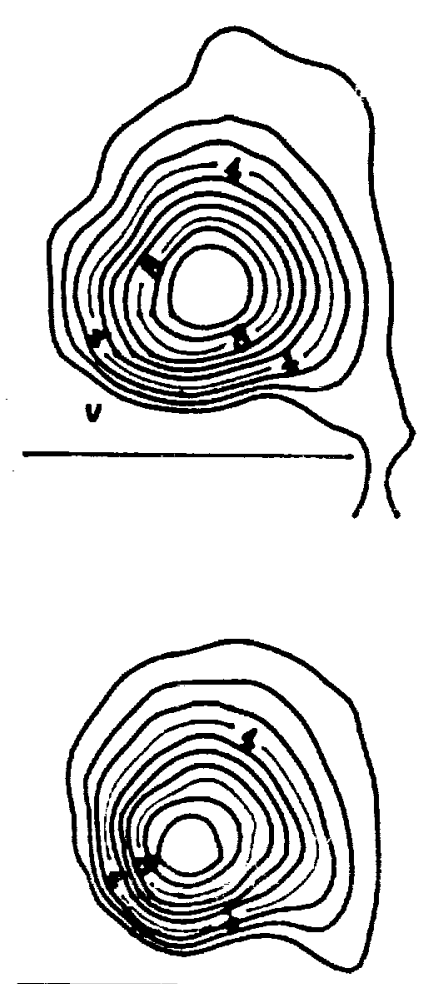

Pitch-up-down
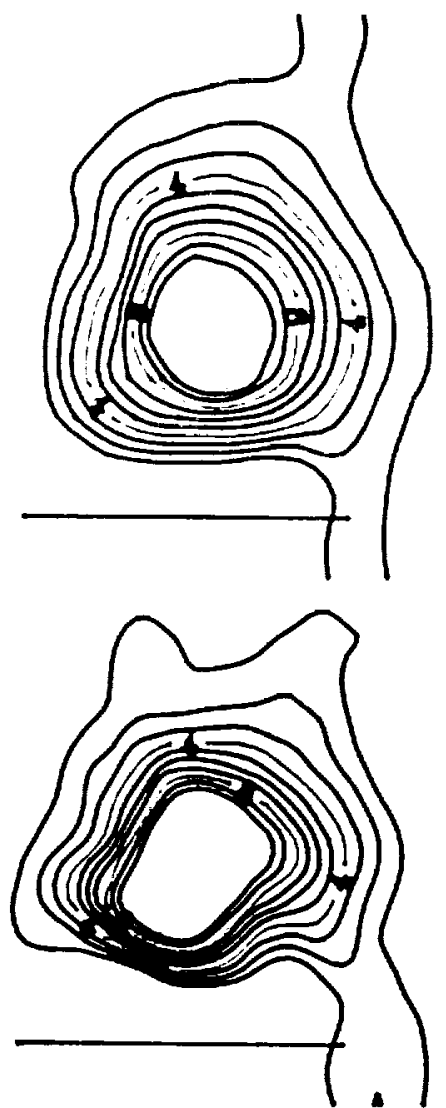

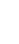




\section{LOCAL CONTROL AT MODERATE TIME SCALES $\mathbf{t U} / \mathrm{C} \sim 1$}

- Response of Leading-Edge Vortex to Time-Dependent Variations of Leading-Edge Separation: Restabilization of Vortex Core

- Response of Leading-Edge Vortex: Structure in CrossFlow Plane During Restabilization in Relation to Separation Conditions

- Response of Leading-Edge Vortex: Variations of Feeding Sheet and Vorticity Distributions of Restabilized Vortex 

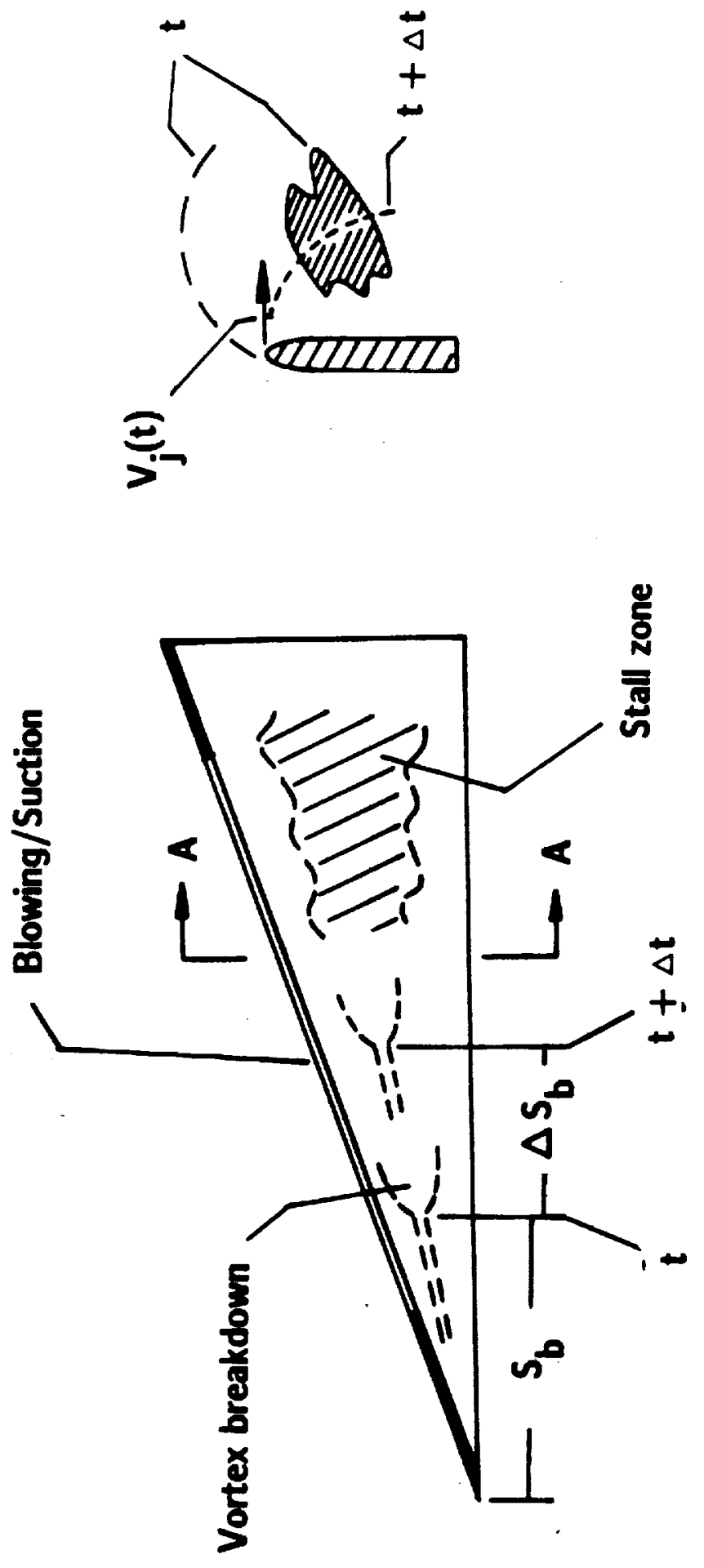